Vierneisel, Carolin; Nitschke, Johannes

\title{
(De-)Professionalisierungstendenzen?! Vielfalts*sensible Bildung im
}

\section{Lehramtsstudium}

Baar, Robert [Hrsg.]; Hartmann, Jutta [Hrsg.]; Kampshoff, Marita [Hrsg.]: Geschlechterreflektierte Professionalisierung. Geschlecht und Professionalität in pädagogischen Berufen. Opladen ; Berlin ; Toronto : Verlag Barbara Budrich 2019, S. 103-118. - (Jahrbuch erziehungswissenschaftliche Geschlechterforschung; 15)

Quellenangabe/ Reference:

Vierneisel, Carolin; Nitschke, Johannes: (De-)Professionalisierungstendenzen?! Vielfalts*sensible Bildung im Lehramtsstudium - In: Baar, Robert [Hrsg.]; Hartmann, Jutta [Hrsg.]; Kampshoff, Marita [Hrsg.]: Geschlechterreflektierte Professionalisierung. Geschlecht und Professionalität in pädagogischen Berufen. Opladen ; Berlin ; Toronto : Verlag Barbara Budrich 2019, S. 103-118 - URN: urn:nbn:de:0111-pedocs-219045 - DOI: 10.25656/01:21904

https://nbn-resolving.org/urn:nbn:de:0111-pedocs-219045

https://doi.org/10.25656/01:21904

in Kooperation mit / in cooperation with:

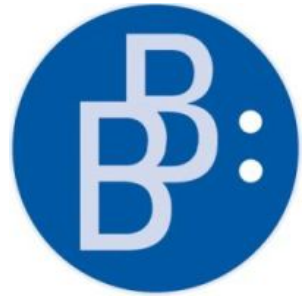

https://www.budrich.de

\section{Nutzungsbedingungen}

Gewährt wird ein nicht exklusives, nicht übertragbares, persönliches und beschränktes Recht auf Nutzung dieses Dokuments. Dieses Dokument is ausschließlich für den persönlichen, nicht-kommerziellen Gebrauch bestimmt. Die Nutzung stellt keine Übertragung des Eigentumsrechts an diesem Dokument dar und gilt vorbehaltlich der folgenden Einschränkungen: Auf sämtlichen Kopien dieses Dokuments müssen alle Urheberrechtshinweise und sonstigen Hinweise auf gesetzlichen Schutz beibehalten werden. Sie dürfen dieses Dokument nicht in irgendeiner Weise abändern, noch dürfen Sie dieses Dokument für öffentliche oder kommerzielle Zwecke vervielfältigen, öffentlich ausstellen, aufführen, vertreiben oder anderweitig nutzen.

Mit der Verwendung dieses Dokuments erkennen Sie die Nutzungsbedingungen an.

\section{Terms of use}

We grant a non-exclusive, non-transferable, individual and limited right to using this document.

This document is solely intended for your personal, non-commercial use. Use of this document does not include any transfer of property rights and it is conditional to the following limitations: All of the copies of this documents must retain all copyright information and other information regarding legal protection. You are not allowed to alter this document in any way, to copy it for public or commercial purposes, to exhibit the document in public, to perform, distribute or otherwise use the document in public.

By using this particular document, you accept the above-stated conditions of use.

\section{Kontakt / Contact:}

\section{peDOCS}

DIPF | Leibniz-Institut für Bildungsforschung und Bildungsinformation Informationszentrum (IZ) Bildung

E-Mail: pedocs@dipf.de

Internet: www.pedocs.de

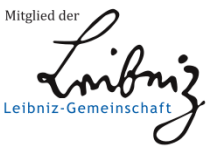


Robert Baar

Jutta Hartmann

Marita Kampshoff (Hrsg.)

\section{$15 / 2019$}

Jahrbuch erziehungswissenschaftliche Geschlechterforschung

Geschlechterreflektierte Professionalisierung Geschlecht und Professionalität in pädagogischen Berufen 


\section{Robert Baar}

Jutta Hartmann

Marita Kampshoff (Hrsg.)

\section{Geschlechterreflektierte Professionalisierung - Geschlecht und Professionalität in pädagogischen Berufen}

Verlag Barbara Budrich Opladen • Berlin • Toronto 2019 
Bibliografische Information der Deutschen Nationalbibliothek

Die Deutsche Nationalbibliothek verzeichnet diese Publikation in der Deutschen

Nationalbibliografie; detaillierte bibliografische Daten sind im Internet über

http://dnb.d-nb.de abrufbar.

Gedruckt auf säurefreiem und alterungsbeständigem Papier.

Alle Rechte vorbehalten.

(C) 2019 Verlag Barbara Budrich, Opladen, Berlin \& Toronto

www.budrich-verlag.de

ISBN 978-3-8474-2277-8 (Paperback)

eISBN 978-3-8474-1310-3 (eBook)

Das Werk einschließlich aller seiner Teile ist urheberrechtlich geschützt. Jede Verwertung außerhalb der engen Grenzen des Urheberrechtsgesetzes ist ohne Zustimmung des Verlages unzulässig und strafbar. Das gilt insbesondere für Vervielfältigungen, Übersetzungen, Mikroverfilmungen und die Einspeicherung und Verarbeitung in elektronischen Systemen.

Typographisches Lektorat: Anja Borkam, Jena

Umschlaggestaltung: disegno visuelle kommunikation, Wuppertal - www.disenjo.de Druck: paper \& tinta, Warschau

Printed in Europe 


\section{Inhalt}

\section{Einleitung}

Robert Baar, Jutta Hartmann \& Marita Kampshoff

Vorwort. 7

Robert Baar, Jürgen Budde, Marita Kampshoff \& Astrid Messerschmidt Redaktion des Jahrbuchs erziehungswissenschaftliche

Geschlechterforschung

Von der Frauen- und Geschlechterforschung in der

Erziehungswissenschaft zur erziehungswissenschaftlichen

Geschlechterforschung....

Maja S. Maier

Erziehungswissenschaftliche Geschlechterforschung?

Ein Essay zu Verhältnisbestimmung und Forschungsprogrammatik 15

Robert Baar, Jutta Hartmann \& Marita Kampshoff

Geschlechterreflektierte Professionalisierung - Geschlecht und

Professionalität in pädagogischen Berufen. Eine Einführung

\section{Vielfältige Lebensweisen im Fokus von Professionalität}

Florian Cristobal Klenk

Interdependente Geschlechtervielfalt als un/be/deutende Anforderung an pädagogische Professionalität

Mart Busche \& Uli Streib-Brzič

Die Entwicklung heteronormativitätskritischer Professionalität in

Reflexions-Workshops - Zur Verbindung von pädagogischem

Erfahrungswissen und wissenschaftlichem Erkenntniswissen im Kontext

von Praxisforschung

Carolin Vierneisel \& Johannes Nitschke

(De-)Professionalisierungstendenzen?! Vielfalts*sensible Bildung im

Lehramtsstudium. 


\section{Geschlechterreflektierte Professionalität im Elementarbereich}

\section{Melanie Kubandt}

Ansprüche an ein geschlechtergerechtes, professionelles Handeln im Elementarbereich - Ethnographische Perspektiven auf Ungewissheiten,

Komplexitäten und Grenzen im pädagogischen Alltag

Susann Fegter, Anna Hontschik, Eszter Kadar, Kim-Patrick Sabla \& Maxine Saborowski

Bezüge auf Familie als Moment der Vergeschlechtlichung pädagogischer Professionalität: Diskursanalytische Perspektiven auf Äußerungen in Gruppendiskussionen mit Kita-Teams

\section{Herausforderungen sexualpädagogischer Professionalisierung}

\section{Anja Eichhorn}

Doing Sexual Agency: Sexuelle Handlungsfähigkeit sexuell

missbrauchter jugendlicher Mädchen in der stationären Jugendhilfe

Marion Thuswald

Geschlechterreflektierte sexuelle Bildung?

Heteronormativität und Verletzbarkeit als Herausforderungen

sexualpädagogischer Professionalisierung

\section{Rezension}

Marina Dangelat, Frauke Grenz \& Christine Thon

Rezension zu: Gesicht Zeigen! (2017) (Hrsg.): „Weiße können nicht rappen“. Das Positionierungsspiel gegen Vorurteile und Klischees und zu: Wedl, Juliette (2018): Identitätenlotto. Ein Spiel quer durchs Leben ... 183

Verzeichnis der Autor_innen 191 
Carolin Vierneisel, Johannes Nitschke

\section{(De-)Professionalisierungstendenzen?! Vielfalts*sensible Bildung im Lehramtsstudium}

\section{Paradoxe Pluralisierungen}

„Schools and colleges of education have been shown, in many cases, to breed deeper heterosexism in teachers - through explicit hostility toward the issue [...] or its omission from teacher education programs." (Gorski, Davis \& Reiter 2013: 225)

Seit den 1990er Jahren rücken Praxen der Thematisierung und Dethematisierung von sexueller und geschlechtlicher Vielfalt im Bildungssystem und die Folgen schulischer Differenz- und Diskriminierungserfahrungen für queere Jugendliche verstärkt in den Blick. Bis heute zeigt sich dabei, wie Schulen häufig einen Ort darstellen, der eine institutionalisierte heteronormative Enkulturation von Jugendlichen organisiert (vgl. Kleiner 2015: 30). Die Daten zu psychosozialen Folgen, ,Coming-Out'-Erfahrungen, Suizidraten als auch Schulbuchuntersuchungen (siehe für einen Überblick bspw. Klocke 2012; Kleiner 2015; Krell \& Oldemeier 2016) verweisen auf ein Gender- und Sexualitätsregime in der Schule (vgl. Jäckle et al. 2016: 23), welches spezifische Wissensordnungen und Subjektpositionierung von Über- und Unterordnung und damit Privilegierung und Deprivilegierung transportiert. Diese Befunde sind in Bezug auf das Bildungswesen problematisch, soll dieses eigentlich die Enkulturation in eine plurale demokratische Gesellschaft ermöglichen (vgl. Huch \& Lücke 2015: 7) und ist doch „Menschenrechtserziehung [...] Aufgabe für den gesamten Unterricht und Aufgabe aller Lehrerinnen und Lehrer" (KMK 2000 [1980]: 5).

Eingelagert werden können diese Befunde in die Theoretisierung grundsätzlicher paradoxaler dominanzkultureller (Rommelspacher 1998) Spannungsfelder, in denen Gleichheitsrhetorik und Ungleichheitsverhältnisse sowie Be- und Verachtung oszillieren. Innerhalb der erziehungswissenschaftlichen Debatte wird das Spannungsverhältnis aus Pluralisierung und Normierung vielfältiger geschlechtlicher und sexueller Lebensweisen in Gesellschaft wie in der Pädagogik seit Anfang der 2000er Jahre thematisiert (vgl. Hartmann 2002). Der Themenbereich sexueller und geschlechtlicher Vielfalt muss daher mit Blick auf die ,widersprüchlichen Gleichzeitigkeiten“(Henningsen, Tuider \& Timmermanns 2016: 7) diskutiert werden. Die scheinbar zunehmende Normalität sexueller und geschlechtlicher Vielfalt steht im Widerspruch zu deren 
Dethematisierung, Abwertung und Stereotypisierung in alltagsweltlichen und schulischen Handlungspraxen (vgl. Schmidt, Schondelmayer \& Schröder 2015: 11). Die in der Pädagogik bereits entwickelten Zugänge zum Themenbereich werden dort kaum aufgegriffen.

Ein pädagogischer Kontext, in dem diese Dynamiken auch erst nach und nach betrachtet werden, ist der des Lehramtsstudiums. Bisher im deutschsprachigen Bereich nicht beachtet ist die Rolle derjenigen, die im Lehramtsstudium dozieren. Es sind Konzepte von Professionalität und Professionalisierung, die eine Möglichkeit eröffnen, paradoxale Spannungsfelder von Anerkennungsrhetorik und Ungleichheitsverhältnissen, von Be- und Verachtung sowie von Entgrenzung und Begrenzung in dominanzkulturellen Verhältnissen - auch im Kontext des Lehramtsstudiums - zu rahmen. Dabei bietet sich ein strukturtheoretischer Professionalisierungsbegriff - verstanden als pädagogisch-professionelles Handeln in paradoxen Verhältnissen (vgl. Helsper 1996: 528) - an, um eine Pädagogik zu skizzieren, die die Anerkennung von Differenz begleitet, vermittelt und organisiert.

„Wenn die Konstituierung, die Wahrung und die Restituierung leiblicher, psychischer, moralischer und sozialer Anerkennung lebenspraktisch bedroht ist, scheitert oder aus eigener Kraft nicht gesichert werden kann oder diese zeitweise gefährdet ist, treten Professionelle zu ihrer Sicherung und Restituierung in Kraft.“ (Helsper 1996: 529)

Dies bedeutet auch, dass Professionalisierung etwas damit zu tun hat, Bedrohungen von Anerkennungsverhältnissen zu erkennen sowie die Verstrickungen in die Produktion von Normalität zu thematisieren.

Der vorliegende Text will zur näheren Bestimmung dieser Dynamiken im Kontext des Lehramtsstudiums beitragen. Ausgehend von der Darstellung (inter-)nationaler Forschungsergebnisse werden Erkenntnisse aus einer empirischen Studie zu sexueller und geschlechtlicher Vielfalt in der Lehre des Lehramtsstudiums vorgestellt. Diese werden anschließend vor einem professionalisierungstheoretischen Rahmen im Kontext des Pluralisierungsparadoxons (vgl. Helsper 1996: 540) und neoliberaler Organisationslogiken eingeordnet.

\section{2 (Un-)Sichtbarkeiten - Ausgangslage im Kontext Lehramtsstudium}

In einer der wenigen deutschsprachigen Studien zur Thematisierung von Geschlecht im Studium der Erziehungs- und Bildungswissenschaften stellt Klinger nach Gruppendiskussionen mit Studierenden fest, dass diese innerhalb des Studiums keine Zugänge zu erziehungswissenschaftlicher Geschlechterfor- 
schung entwickelt haben und „die eigene vergeschlechtlichte und vergeschlechtlichende Position von den Studierenden nicht hinterfragt wird" (Klinger 2014: 339). Dies verwundert nicht, haben doch nur $14 \%$ der Standorte, die Erziehungswissenschaften anbieten, Professuren mit einer Denomination, die auf eine institutionelle Verankerung der pädagogischen Geschlechterforschung verweisen, sodass nicht davon ausgegangen werden kann, ,dass es sich um gesicherte Formen für die Weitergabe relevanten Wissens [der Geschlechterforschung] handelt" (Faulstich-Wieland \& Wesseloh 2016: 204).

Eine naheliegende, jedoch bisher selten formulierte Forderung als Konsequenz der skizzierten Erfahrungen vieler queerer Jugendlicher an Schulen ist die stärkere Fokussierung derjenigen, die die zukünftigen Lehrkräfte belehren: deren Dozierende (vgl. Mills \& Ballantyne 2016: 275). Jenseits des Lehramtsstudiums lässt eine der wenigen Studien (Bütow, Eckert \& Teichmann 2016) zu Hochschuldozierenden und Gender im deutschsprachigen Kontext die Nebelfelder erahnen, die es als Akteur_in im Feld Gender/sexuelle und geschlechtliche Vielfalt in der Lehre bzw. Hochschuldidaktik zu erschließen gilt. Ausgehend von ihren Analysen konstatieren die Autor_innen eine geringe zugeschriebene Bedeutung von Gender als Lehrinhalt bei den Dozierenden in den unterschiedlichen Fachdisziplinen. Dies äußere sich zum einen darin, dass eine Themenverantwortlichkeit divers anderen zugeschrieben werde, wenn z. B. keine Berührungspunkte von Genderaspekten zur eigenen Lehre gesehen würden, Vertretende der Naturwissenschaften eher die Kolleg_innen der Sozialwissenschaften in der Pflicht sähen oder Menschen die Beschäftigung mit Genderfragen als Expert innen- und Frauendiskurs abspeicherten. In den Ergebnissen zeigen sich aber auch zwei weitere Phänomene: zum einen eine klar ablehnende Haltung gegenüber der Integration von Gender in die eigene Lehre; zum anderen die Bedeutung sozialer Erwünschtheit in diesem Diskurs, wenn ein Desinteresse an dem Thema bei Kolleg_innen nur vermutet werden kann und wird, da ein offener Austausch aufgrund eines wahrgenommenen Klimas der ,political correctness“ (Bütow, Eckert \& Teichmann 2016: 76, Herv. i. O.) als unmöglich beschrieben wird.

Zurück im Kontext Lehramtsstudium können Ferfolja und Robinson (2004: 13) zeigen, dass Dozierende der ,anti-homophobia-education“ in der Schule zwar eine gewisse Bedeutung zumessen - andere Dimensionen sozialer Gerechtigkeit von ihnen meist aber als bedeutender erachtet werden. Dieses Ergebnis wird für den deutschsprachigen Kontext durch den Abschlussbericht des Forschungsprojektes LeWi (Ihsen 2013) gestützt, der aufführt, wie interviewte Hochschuldozierende Vielfalt und Diversity meist mit der Differenzkategorie Migration(-serfahrung) gleichsetzen. Nach Ferfolja und Robinson (2004: 14) wird eine Bedeutung von sexueller und geschlechtlicher Vielfalt häufig überhaupt erst für spätere Schulphasen zuerkannt und im Fall der Grundschule abgesprochen. Antihomophobe Arbeit wird durch die Befragten dieser Studie häufig als Zusatzaufgabe und nicht als integraler Bestandteil des 
Lehrauftrages verstanden. Bei der Frage nach Faktoren, die solche Relevanzsetzungen beeinflussen, identifizieren Smolen et al. (2006: 54) in einem Sample von Mitgliedern einer lehramtsbildenden Fakultät unter anderem die Bedeutung des Kriteriums des Standortes, also vermutlich auch Aspekte einer wahrgenommenen Offenheit des Kollegiums gegenüber dem Thema. Ein weiteres Problemfeld benennt die Studie durch die festgestellte Diskrepanz zwischen der durch die Fakultätsmitglieder angegebenen Unterstützung der Ziele von Diversity Trainings und der Verbindlichkeit in deren Umsetzung im eigenen Arbeitsfeld.

In mehreren Studien werden in diesem Zusammenhang häufig so genannte (persönliche) Risiken thematisiert, die Dozierende und Studierende des Lehramtsstudiums mit einer Thematisierung sexueller und geschlechtlicher Vielfalt einzugehen fürchten (vgl. Ferfolja \& Robinson 2004). Darunter fallen Sorgen, (Schul-)Kinder zu überfordern oder in ihrer sexuellen Orientierung zu beeinflussen (ebd.), die Befürchtung, als lehrende Person selbst für lesbisch, schwul, bisexuell, trans*- oder intergeschlechtlich gehalten $\mathrm{zu}$ werden bis hin zur Angst vor rechtlichen Konsequenzen (vgl. Nzimande 2015: 77). Auch können Bedenken mitschwingen, nicht den Haltungen und Wünschen aller Studierender in diesem Themenbereich gerecht oder gar für homophob gehalten zu werden, wenn Dozierende selbst z. B. aus Unwissenheit oder fehlender Reflexion Stereotype reproduzieren (vgl. Nzimande 2015: 77). Selbst erfahrenen Trainer_innen und Dozierenden im Bereich sozialer Gerechtigkeit gelingt es demnach nicht immer, heteronormativitäts-affirmative Aussagen oder Handlungen zu vermeiden (vgl. Schmidt et al. 2012: 1179). Wichtig an dieser Stelle scheint es, die Grenzen bzw. die Ungewissheit pädagogischer Interventionen anzuerkennen und realistische Ziele im Bildungsprozess von Lehramtsstudierenden zu wählen: So ist es nach Villegas und Lucas (2002: 30) unrealistisch zu erreichen, dass Studierende am Ende ihres Studiums über ein ausgefeiltes und erprobtes Repertoire an Handlungskonzepten in Bezug auf sexuelle und geschlechtliche Vielfalt in der Schulpraxis verfügen. Ziel einer Lehre solle es vielmehr sein, dass die Studierenden während des Studiums eine Vision von sich als Lehrkraft entwickeln, die sexuelle und geschlechtliche Vielfalt als bedeutsam erachtet, und dass sie bis dato Gelerntes als Orientierungsrahmen nutzen, dieser Vision Schritt für Schritt in ihrem Lehralltag an Schulen näher zu kommen.

Eine weitere, bei Dozierenden identifizierte Hürde für die Thematisierung sexueller und geschlechtlicher Vielfalt in der Lehre betrifft erkenntnistheoretische Überzeugungen bzw. den Aspekt „Lehren mit Epistemologie“ (Eckert 2014: 47). So zeigt sich z. B. ein für den universitären Kontext oft beschriebener Glaube an objektives Wissen - im Sinne einer Wertefreiheit in Lehre und Forschung - als kaum vereinbar mit einer kritischen Pädagogik, die gesellschaftliche Machtverhältnisse hinterfragt und häufig als Grundlage für Trai- 
nings zu sexueller und geschlechtlicher Vielfalt herangezogen wird (vgl. Nzimande 2015: 78). Neben der Vorstellung eines objektiven und wertfreien Wissens ist es auch der Glaube an das Primat des Wissens als höchste Form des Erkenntnisgewinns, den es zu hinterfragen gilt. Anlass des Zweifels sind der humanistischen Tradition verschriebene Trainings, die mit dem Ansatz der Förderung von Toleranz und Akzeptanz durch Wissensvermittlung nur eingeschränkt Erfolge in der Reduzierung von Vorurteilen und Diskriminierung erzielen (vgl. Ferfolja \& Robinson 2004: 20). Alternativ zeigen Umsetzungen in der Praxis auf, wie es neben einer Wissensvermittlung Räume für die Reflexion bestehender Machtverhältnisse und eigener Verwicklungen darin bedarf (vgl. Ferfolja \& Robinson 2004; Robinson \& Ferfolja 2008; Nzimande 2015). Räume der Reflexion sollen auch dem Problem begegnen, dass Lehrende soziale Gerechtigkeit auf der Ebene des Individuums verhandeln und darüber die Bedeutung gesellschaftlicher Machtverhältnisse sowie deren Veränderung auBen vor lassen.

\section{Empirischer Impuls - eine Studie zu Vielfalt* in der Lehre}

\subsection{Methoden}

Die hier dargestellte quantitative Untersuchung fand im Rahmen des Projektes „Vielfalt Lehren!“1 statt, das auf eine Förderung der Auseinandersetzung mit sexueller und geschlechtlicher Vielfalt (kurz: Vielfalt*) bei Dozierenden im Lehramtsstudium an der Universität Leipzig abzielt. Die hier dargestellten Ergebnisse beziehen sich auf ein Subsample der Universität Leipzig (UL) ${ }^{2}$. Entsprechend der Zielsetzung des Projektes ist die quantitative Untersuchung im Feld der Aktionsforschung (vgl. Unger, Block \& Wright 2007) zu verorten.

Die quantitative Untersuchung erhebt bei Dozierenden des Lehramtsstudiums Daten in den Bereichen Wissen, Erfahrungen, Relevanzsetzungen, Her-

1 „Vielfalt Lehren!“ ist ein Kooperationsprojekt der Professur Schulpädagogik/Schulentwicklungsforschung, dem Zentrum für Frauen- und Geschlechterforschung der Universität Leipzig und der Akademie Waldschlösschen im Rahmen des Modellprojekts „Akzeptanz für Vielfalt - gegen Homo-, Trans*- und Inter*feindlichkeit“ im Bundesprogramm „Demokratie Leben" des Bundesministerium für Familie, Senioren, Frauen und Jugend.

2 Die quantitative Erhebung fand 2018 zudem an den Technischen Universitäten Chemnitz und Dresden und der Carl-von-Ossietzky-Universität Oldenburg statt. 
ausforderungen und Bedarfe zu Vielfalt* in der Lehre. Der Fragebogenerstellung ging eine Recherche bestehender Erhebungsinstrumente in dem Feld Vielfalt* voraus - für den Bereich der Herausforderungen wurden Items aus dem Instrument von Ulrich Klocke (2012) zur Erhebung der Akzeptanz sexueller und geschlechtlicher Vielfalt an Berliner Schulen übernommen und angepasst. Die weitere Erstellung erfolgte insofern partizipativ, als Mitglieder des Projekt-/Praxisbeirates daran beteiligt waren, die z. T. selbst im Lehramtsstudium oder an Schulen lehren. Die Erhebung fand im Januar 2018 über zweieinhalb Wochen statt.

Die Ambivalenzen bei der Verwendung quantitativer Verfahren in heteronormativitätskritischer Forschung - die Trivialisierung komplexer gesellschaftlicher Verhältnisse und die Reproduktion kategorialer Zuschreibungen (vgl. Voß 2011: 7) - konnten auch in dieser Erhebung nicht umgangen werden, wurden aber reflektiert.

\subsection{Sample}

Zwei Drittel (66,9\%) der insgesamt 138 Befragten geben ein Alter von unter 40 Jahren an, sodass sich ein Bild von einem vergleichsweise jungen Sample an universitär Beschäftigten ergibt ${ }^{3}$. Die Teilnehmenden ordnen sich selbst den Kategorien weiblich (52,3 \%), männlich (46,1\%) und keine Geschlechtszugehörigkeit $(1,6 \%, n=2) \mathrm{zu}^{4}$. Weitere Geschlechtszugehörigkeiten wurden erfragt, aber nicht angegeben. Mit einem Anteil von 17,5\% bezeichnet sich ca. jede sechste Person als lesbisch, bisexuell, schwul oder queer ${ }^{5}$. Es beteiligten sich mit einer großen Mehrheit wissenschaftliche Mitarbeiter_innen (50\%) und Lehrkräfte für besondere Aufgaben (26,9 \%) an der Studie - Professor_innen waren mit 19,2\% vertreten. Mit 40,2\% verortet sich die größte Gruppe der Befragten innerhalb des Lehramtsstudiums bei den Fachwissenschaften. Mitarbeiter_innen der Studiengänge (bspw. Lehramt an Grundschulen oder Oberschulen), Ergänzungsstudien und der Förderpädagogik sind mit 28,8 \%

3 Der Median der unbefristet an der Universität Leipzig beschäftigten wissenschaftlichen Mitarbeitenden liegt zwischen 50 und 55 Jahren. Quelle: https://www.statistik.sachsen.de/download/100_Berichte-B/B_III_4_j16_SN.pdf Letzter Zugriff: 21.02.2018.

4 An der Universität Leipzig zeigt sich ein Verhältnis der erhobenen Kategorien von 44,4 \% (Frauen) zu 55,6 \% (Männer). Quelle: https://www.statistik.sachsen.de/download/100_Berichte-B/B III 4 j16 SN.pdf Letzter Zugriff: 21.02.2018.

5 Eine weitere Person, die sich „keiner sexuellen Orientierung“ zuordnete, ist weiterhin in der Gruppe eingeschlossen. Es wurden keine weiteren sexuellen Orientierungen genannt. 
vertreten und Angehörige der Fachdidaktik mit 12,1\%; dabei arbeitet allerdings fast jede fünfte befragte Person in mehr als einem dieser Bereiche $(18,9 \%){ }^{6}$

Während eine große Mehrheit von 93,5 \% durch ihr persönliches, berufliches oder erweitertes Umfeld lesbische, schwule, bisexuelle, trans*- oder intergeschlechtliche Menschen kennt, ist das bei neun Befragten (6,5\%) nicht der Fall. Gleichzeitig haben sich nach eigener Auskunft fünf Personen $(3,6 \%)$ bisher noch nie mit Vielfalt* auseinandergesetzt. Die öffentliche Thematisierung von Vielfalt* halten $86,3 \%$ für wichtig und im Umfang angemessen bzw. ausbaubar. Andererseits ordnen 13,7\% die Thematisierung als unwichtig ein, da sie bereits zu viel Raum einnehme bzw. gar keinen einnehmen solle. Dass Studierende im Lehramtsstudium lernen, Vielfalt* in der Schule zu thematisieren, hält jede fünfte Person für eher oder sehr unwichtig (19,2\%). Elf Befragte $(8 \%)$ haben in ihren Lehrveranstaltungen noch nie eine lesbische, schwule, bisexuelle, trans*- oder intergeschlechtliche Person wahrgenommen. Sechs Befragte $(4,3 \%)$ haben Vorfälle von Diskriminierung aufgrund der sexuellen Orientierung oder Geschlechtszugehörigkeit wahrgenommen.

\subsection{Vielfalt in der Lehre: Relevanzsetzungen, Wissen und Umsetzungen}

Den Schwerpunkt unserer Betrachtungen bildet zunächst die Entwicklung des Antwortverhaltens der Befragten von Relevanzsetzungen über Wissen hin zu Umsetzungen in Bezug auf Vielfalt* in (eigenen) Lehrveranstaltungen des Lehramtsstudiums (vgl. Tabelle 1). Im Vergleich zur angegebenen hohen Relevanz der Thematisierung von Vielfalt* in der allgemeinen Öffentlichkeit (86,3\%), erachten es nur drei Viertel der Befragten (74,6 \%) als sehr oder eher wichtig, dass Vielfalt* als Lehrinhalt thematisiert wird. Die Berücksichtigung von Vielfalt* in der Interaktion mit Studierenden sehen noch $71,2 \%$ als relevant an, die Berücksichtigung von Vielfalt* bei der Vermittlung der Lehrinhalte sind noch für zwei Drittel aller Befragten $(66,7 \%)$ bedeutsam.

Eine Fortschreibung dieses Antwortverhaltens zeigt sich im Bereich des eigenen Wissens. Eigenes Wissen zu Vielfalt* in der Lehre sehen die Befragten noch im Bereich der Interaktion mit Studierenden ausgebildet: Hier sagen $31,8 \%$, dass sie voll und ganz wissen, wie sie vielfalts*sensibel mit Studierenden interagieren können, $54,3 \%$ wissen es eher, während das bei $14 \%$ eher

6 Die erziehungswissenschaftliche Fakultät an der UL bietet Lehramts- und Masterstudiengänge an. Dozierende, die in Studiengängen, Ergänzungsstudien und der Förderpädagogik lehren, sind in der Regel an der erziehungswissenschaftlichen Fakultät angesiedelt. 
Tabelle 1: Relevanzsetzungen, Wissen und Umsetzungen vielfalts*sensibler Lehre

\begin{tabular}{ccccc}
\hline & \multicolumn{4}{c}{ Angaben in \% } \\
\hline Relevanz von Vielfalt* .. & Sehr wichtig & Eher wichtig & Eher unwichtig & Sehr unwichtig \\
$\ldots$ als Lehrinhalt $(\mathrm{n}=130)$ & 33,1 & 41,5 & 19,2 & 6,2 \\
$\ldots$ in der Vermittlung $(\mathrm{n}=132)$ & 34,8 & 31,8 & 26,5 & 6,8 \\
$\ldots$ in der Interaktion $(\mathrm{n}=132)$ & 40,2 & 31,1 & 22,7 & 6,1 \\
\hline Wissen zu Integration von Vielfalt* & Voll und ganz & Eher vorhanden & Eher nicht & Gar nicht \\
& vorhanden & vorhanden & vorhanden \\
$\ldots$ als Lehrinhalt $(\mathrm{n}=133)$ & 18,8 & 46,6 & 27,8 & 6,8 \\
$\ldots$ in der Vermittlung $(\mathrm{n}=130)$ & 16,2 & 45,4 & 35,4 & 3,1 \\
$\ldots$ in der Interaktion $(\mathrm{n}=129)$ & 31,8 & 54,3 & 12,4 & 1,6 \\
\hline Umsetzungen von Vielfalt* ... & Regelmäßige & Eine/Einige & Keine Umsetz- & Keine Umsetz- \\
Umsetzungen & Umsetzungen & ungen bisher & ungen geplant \\
... als Lehrinhalt $(\mathrm{n}=134)$ & 20,1 & 41,8 & 20,9 & 17,2 \\
$\ldots$ in der Vermittlung $(\mathrm{n}=128)$ & 15,6 & 31,3 & 35,9 & 17,2 \\
... in der Interaktion $(\mathrm{n}=130)$ & 43,1 & 30,0 & 16,9 & 10,0 \\
\hline
\end{tabular}

Quelle: Eigene Darstellung

oder gar nicht der Fall ist. Noch $18 \%$ der Befragten wissen voll und ganz, wie sie in ihrer Lehre Vielfalt* als Inhalt thematisieren können, 46,6 \% wissen es eher, während jede dritte Person (34,6 \%) angibt, eher kein oder gar kein Wissen dazu zu besitzen. Am wenigsten stark ausgeprägtes Wissen gibt es in der Selbsteinschätzung mit 16,2\% dazu, wie die Vermittlung von allgemeinen Lehrinhalten vielfalts*sensibel gestaltet werden könne (eher: 45,4\%; eher nicht/gar nicht: 38,5\%).

Eine weitere Reduktion ist bei den erfolgten Umsetzungen zu verzeichnen - gleichwohl findet sich die gleiche Dynamik wie bei den Wissenszuschreibungen: So gibt bei dem Aspekt der Berücksichtigung von Vielfalt* in der Interaktion mit Studierenden mit fast drei Viertel der größte Teil der Befragten $(73,1 \%)$ an, diese bereits umgesetzt zu haben. Ebenso eine Mehrheit $(61,9 \%)$ hat bereits Vielfalt* als Lehrinhalt thematisiert (vs. 38,1\%, die das nicht taten). Schlusslicht bildet wieder die vielfalts*sensible Vermittlung von Lehrinhalten, bei der die Mehrheit der Befragten angibt, dies noch nie umgesetzt zu haben (ja: 46,9 \%, nein: 53,1 \%). In den Fällen, in den es zu Umsetzungen bezüglich der drei Aspekte kommt, berichten die befragten Dozierenden überwiegend von sehr guten und eher guten Erfahrungen (Sehr und eher gute Erfahrungen in den Bereichen: Vielfalt* als Lehrinhalt, 81,0\%; Vielfalt* in der Vermittlung, 86,8 \%; Vielfalt* in der Interaktion: 83,1\%).

Der Rahmung des Professionalisierungsdiskurses folgend, lohnt es sich, die Organisation Universität und dessen Statusgruppen im Lehramtsstudium in den Blick zu nehmen. So betrachten Professor_innen durchgängig die Thema- 
tisierung von Lehrinhalten, die vielfalts*sensible Vermittlung und eine vielfalts*sensible Interaktion als weniger wichtig als wissenschaftliche Mitarbeiter_innen und Lehrkräfte für besondere Aufgaben. Gleichzeitig geben sie aber zum einen häufiger an, über Wissen zu den Themenkomplexen zu verfügen und zum anderen, diese Aspekte in ihrer Lehre umgesetzt zu haben (vgl. Tabelle 2).

Tabelle 2: Relevanz, Wissen und Umsetzungen vielfalts*sensibler Lehre nach Position

\begin{tabular}{cccc}
\hline & \multicolumn{2}{c}{ Zustimmung in $\%$} & \\
& Professor_innen & WiMi/LfbA & Signifikanz \\
\hline Zugeschriebene Relevanz & & & \\
Lehrinhalt $(\mathrm{n}=119)$ & 62,5 & 77,9 & n.s \\
Vermittlung $(\mathrm{n}=119)$ & 56,5 & 67,7 & n.s \\
Interaktion $(\mathrm{n}=120)$ & 64,0 & 75,8 & n.s \\
\hline Vorhandenes Wissen & & & \\
Lehrinhalt $(\mathrm{n}=120)$ & 87,5 & 58,3 & n.s. \\
Vermittlung $(\mathrm{n}=118)$ & 73,9 & 57,9 & n.s. \\
Interaktion $(\mathrm{n}=117)$ & 95,5 & 84,2 & \\
\hline Erfolgte Umsetzungen & & & n.s. \\
Lehrinhalt $(\mathrm{n}=122)$ & 72,0 & 59,8 & n.s. \\
Vermittlung $(\mathrm{n}=117)$ & 58,3 & 43,0 & n.s. \\
\hline Interaktion $(\mathrm{n}=118)$ & 75,0 & 73,4 &
\end{tabular}

Quelle: Eigene Darstellung

\subsection{Der Weg zur Umsetzung: Strukturelle Hindernisse und Fortbildungsbedarfe}

In Bezug auf Hindernisse und Unterstützungsquellen zeigt sich ein Mix an Faktoren relevant: Die Aussage, inhaltliche Fragen Studierender im Themenkomplex Vielfalt* nicht beantworten zu können, bekommt mit 23,3\% die meiste Zustimmung. Aber auch die Ablehnung der Thematisierung von Vielfalt* durch Studierende beschäftigt noch jede zehnte befragte Person $(10,5 \%)$. Während eine Sorge struktureller Art, dass die Thematisierung durch die Leitung abgelehnt wird, kaum geäußert wird, verdeutlichen die Daten gleichermaßen ein Fehlen der Thematisierung durch eben diese Leitung: Nur jede fünfte Person berichtet davon, dass Vielfalt* durch Vorgesetzte thematisiert wurde, während v. a. Kolleg_innen (63,8 \%) aber auch Studierende (60,9\%) Vielfalt* deutlich häufiger ansprechen. Die Bedeutung dieser Faktoren für die (Nicht)- 
Integration von Vielfalt* in der Lehre - ob als Lehrinhalt oder in dessen vielfalts*sensiblen Vermittlung - zeigen folgende signifikante Zusammenhänge: Es finden sich durchgängig signifikante Zusammenhänge zwischen der Thematisierung von Vielfalt* durch Studierende und deren Berücksichtigung auf der Relevanz-, Wissens-, und Handlungsebene: So nehmen z. B. Dozierende, deren Studierende Vielfalt* bereits thematisierten, vielfalts*sensible Vermittlung als relevanter wahr $\left(\chi^{2}=9,2 ; \mathrm{df}=1 ; \mathrm{p}=, 01\right)$ und haben diese häufiger umgesetzt $\left(\chi^{2}=10,68 ; \mathrm{df}=1, \mathrm{p}=, 001\right)$ als Dozierende, deren Studierende das nicht taten. Geringfügig weniger signifikante Zusammenhänge zeigen sich zwischen der Berücksichtigung von Vielfalt* und deren Thematisierung durch Kolleg_innen. ${ }^{7}$ Die Thematisierung durch Vertretende der Leitungsebene zeigt hingegen nur wenige signifikante Zusammenhänge zu Wissen oder Berücksichtigung von Vielfalt* in der Lehre ${ }^{8}$ - ggf. nicht zuletzt dadurch, dass diese Vielfalt* insgesamt seltener thematisieren.

Im Bereich Fortbildungen zu Vielfalt* geben $41,4 \%$ der Befragten an, dass ihnen weder zeitliche noch monetäre Ressourcen zur Verfügung stehen, um ein solches Angebot wahrzunehmen. Während 13,5 \% der Befragten solche Ressourcen beanspruchen können, ist es bei dem größten Teil der Befragten $(45,1 \%)$ so, dass sie hierzu keine Informationen haben. Allerdings kennt lediglich jede fünfte Person (20\%) mindestens ein (Fortbildungs-)Angebot im Bereich Vielfalt* (in der Lehre) - 15 Personen davon universitätsinterne und zehn außeruniversitäre Angebote. Von den 138 befragten Personen äußern zwölf $(8,7 \%)$, schon einmal an einem Angebot zu Vielfalt* allgemein und acht $(4,3 \%)$ an einem Angebot zu Vielfalt in der Lehre teilgenommen zu haben. In der Befragung zeigt sich ein sehr uneinheitlich ausgeprägtes Interesse an (weiteren) Fortbildungen zu Vielfalt*: Während 11,8 \% sehr und 36,2 \% eher daran interessiert sind, ist das bei 38,6 \% eher nicht und bei 13,4 \% gar nicht der Fall.

\subsection{Einordnung und Diskussion der Ergebnisse}

Der Anteil derjenigen, die persönlichen Kontakt zu lesbischen, schwulen, bisexuellen, trans*- und intergeschlechtlichen Menschen angeben, liegt in diesem Sample der Lehramtsdozierenden mit 93,5 \% deutlich höher als zuletzt in

7 So geben die Befragten, die in der Interaktion mit Studierenden Vielfalt* berücksichtigen, signifikant häufiger an, dass von ihren Kolleg_innen Vielfalt* thematisiert wurde $\left(\chi^{2}=9,91\right.$; $\mathrm{df}=1, \mathrm{p}=, 01)$.

8 So geben z. B. diejenigen, bei denen Vielfalt* durch eine Leitungsperson thematisiert wurde, signifikant häufiger an, dass ihnen vielfalts*sensible Vermittlung von Lehrinhalten wichtig ist $\left(\chi^{2}=4,85 ; \mathrm{df}=1, \mathrm{p}=, 05\right)$. 
anderen Studien für die Allgemeinbevölkerung festgestellt wurde (vgl. Küpper, Klocke \& Hoffmann, 2017 ${ }^{9}$ ). Der Anteil derjenigen, die Vielfalt* bereits zu häufig in den Medien thematisiert sehen bzw. es gar nicht thematisiert sehen wollen, ist mit $13,7 \%$ dahingegen deutlich niedriger als in thematisch vergleichbaren Erhebungen (ebd.). Dies könnte zum einen als eine höhere Vielfalts*sensibilität der universitär Beschäftigten gedeutet werden - zum anderen ist eine bei Bütow, Eckert und Teichmann (2016) für diese Gruppe beschriebene, stärker ausgeprägt erscheinende Orientierung an sozialer Erwünschtheit zu berücksichtigen. Es ist weiterhin zu beobachten, dass die Unterstützung der Thematisierung von Vielfalt* kontinuierlich fällt, je konkreter Bezug zu der eigenen Lebens- bzw. Arbeitswelt und damit dem Handlungs- und Aufgabenrepertoire der Befragten genommen wird - ein Hinweis darauf, dass die widersprüchliche Gleichzeitigkeit von Toleranzbekundung und Dethematisierung von Vielfalt* in der Handlungspraxis, wie bereits bei Bütow, Eckert und Teichmann (2016) oder Klocke (2012) nachgezeichnet, auch im universitären Kontext vorherrscht.

Anhand der Ergebnisse lassen sich anstehende Aufgaben auf dem Weg zu einer vielfalts*bewussteren Hochschullehre auf unterschiedlichen Ebenen individuell, sozial und strukturell - verorten, hier konkret auf Ebene der Wahrnehmung, der Thematisierungen und der Repräsentation bzw. Sichtbarkeit.

So scheint die Wahrnehmung von Diskriminierung in diesem Sample relativ niedrig ausgeprägt (4,3\%), wenn sie verglichen wird mit unveröffentlichten Daten des Gleichstellungsbüros zu Diskriminierungserfahrungen an der Universität Leipzig ${ }^{10}$ oder Ergebnissen der Universität Kiel (vgl. Klein \& Rebitzer 2012). Sinnvoll erscheint es, eine Sensibilisierung der Wahrnehmung von Dozierenden in Bezug auf (unbeabsichtigt) diskriminierende Vorfälle verstärkt in (Fortbildungs-)Angebote zu integrieren.

Ein interessanter Ansatzpunkt zeigt sich in der sozialen Interaktion: Die bereits erwähnte, themenbezogene soziale Erwünschtheit, aber vor allem die signifikanten Zusammenhänge zwischen der Thematisierung von Vielfalt* im beruflichen Umfeld, d. h. durch Studierende und Kolleg innen, mit dem Ausmaß der Integration des Themas in die eigene Lehre, geben einen Hinweis auf den möglichen Einfluss interaktionaler Dynamiken. Kolleg innen und Studierende können demnach stärker als bisher als Schlüsselpersonen verstanden werden, über die Lehrende adressiert und für die Bedeutung von Vielfalt* in der Lehre sensibilisiert werden können. In der Konzeption von (Fortbildungs-)Angeboten könnte sich dies z. B. in deren stärker partizipativ oder peerlearning-basierten Ausrichtung widerspiegeln.

Die geringere Thematisierung von Vielfalt* durch Professor innen als Vertretende der Leitungsebene gegenüber Mitarbeiter_innen mag angesichts

9 In der genannten Studie wird nur nach Kontakt zu homo- und bisexuellen Menschen gefragt.

10 Präsentiert durch den Gleichstellungsbeauftragten der UL bei einer universitätsinternen Veranstaltung am 06.10.2017. 
der zaghaften Verbreitung pädagogischer Geschlechterforschung an erziehungswissenschaftlichen Fakultäten nicht verwundern. Gleichzeitig ist sie eine vertane Chance, wenn z. B. fast die Hälfte der Befragten nicht wissen, ob ihnen Gelder und Arbeitszeit zur Verfügung stehen, um Fortbildungen im Bereich Vielfalt* zu besuchen.

\section{Fazit-professionstheoretische Ausblicke}

„If all space is closeted, then perhaps the space of the academy is among the most closeted of them all [...]." (Bell \& Valentine 1995: 24)

Sowohl die im Beitrag diskutierten Ergebnisse anderer Studien als auch die Studie an der Universität Leipzig rufen unter professionstheoretischer Perspektive ambivalente Bilder hervor. Der attestierte Nebel konnte auch hier nicht gelüftet werden, zu paradox gelagert scheinen Rationalisierungs- und Pluralisierungstendenzen, die das Handeln von Dozierenden an Universitäten und deren Umgang mit Differenz, Irritationen und der Komplexität sozialer Realität rahmen.

Der Befund, dass eine Weitergabe relevanten Wissens der erziehungswissenschaftlichen Geschlechterforschung an der Universität nicht gelingt bzw. nicht angemessen organisiert werden kann (vgl. Faulstich-Wieland \& Wesseloh 2016: 204), zeigt sich auch in der vorgestellten Studie deutlich. Gerade die Diskrepanz zwischen der zugemessenen Bedeutung von Vielfalt* und dem selbst attestierten Wissen darum einerseits und den nicht gleichermaßen erfolgenden Berücksichtigung in der Lehrpraxis andererseits, kann als eine Tendenz zur pädagogischen Deprofessionalisierung gelesen werden.

Eine besondere Form der Diskrepanz zwischen Wissen und Handlungspraxis, die sich gleichfalls im Rahmen eines Deprofessionalisierungsdiskurses deuten lässt, wird in den Daten in Bezug auf die Leitungsebene sichtbar. Obwohl Professor_innen angeben, Wissen um Vielfalt* zu haben, sowie größtenteils bejahen dieses in der eigenen Lehre umzusetzen, berichtet nur $1 / 5 \mathrm{der} \mathrm{Be}-$ fragten, dass eben diese Vorgesetzten Vielfalt* (in der Lehre) ihnen gegenüber thematisiert hätten. Die Setzung relevanter Themen durch Leitungspersonen, verstanden als Form bzw. Sicherung der Professionalisierung, findet hier folglich nicht oder nur kaum statt.

Die Leitungstätigkeit von Professor innen ist dabei in komplexe Organisationsprozesse der Universität eingebunden, die einen kurzen Blick auf vorherrschende institutionelle Organisationslogiken nahe legen. Aktuelle Tendenzen verweisen auf eine Ökonomisierung der Universität bzw. auf eine neoliberale Reformstrategie einer auf ,Produktivität‘ und ,Exzellenz ${ }^{‘}$ ausgerichteten Uni- 
versität. Diese Perspektive wird auch in aktuellen Diskursen zu Professionalität/Professionalisierung aufgegriffen, die sich mit Prozessen der Standardisierung und der zunehmenden Marktförmigkeit von Bildung auseinandersetzen (vgl. Helsper \& Tippelt 2011: 278-279). Gerade die Standardisierung erziehungswissenschaftlicher Bildungsinhalte und Prüfungsformen im Lehramtsstudium birgt dabei die Gefahr, die Prozesse der Dethematisierung von Vielfalt* und die damit einhergehenden Deprofessionalisierungstendenzen zu unterstützen, vor allem wenn Analysen einer dekonstruktiven Pädagogik (vgl. Fritzsche et al. 2001) und erziehungswissenschaftlichen Geschlechterforschung nicht als Teil des etablierten Kanons gelten und Dozierende aufgrund standardisierter Inhalte und Prüfungen gar keine Möglichkeiten sehen, Seminar- und Vorlesungsinhalte anzupassen. Die aktuelle Tendenz, geschlechterreflektierende Professionalität angesichts dieser komplexen Umstände innerhalb pädagogischer Bildungseinrichtungen anhand von Kompetenzmodellen zu theoretisieren, greift daher zu kurz. Professionalität als individuellen Erwerb von Kompetenzen zu begreifen, individualisiert das Pädagogische als Interaktion zwischen Kompetenz-Habenden und Lernenden und löst damit Pädagogik von der Analyse und Reflexion gesellschaftlicher Prozesse. Dies „forciert die Trivialisierung der klassischen Idee der Selbstbildung und Selbsttransformation“" (Pongratz 2013: 162). Daher bleibt es notwendig, auch die Universität als Raum der paradoxen Pluralisierungen - als Möglichkeitsraum der kritischen Selbstbildung und „Brutstätte des Heterosexismus“ (Gorski, Davis \& Reiter 2013: 225) - weiterhin in den Blick zu nehmen.

\section{Literatur}

Bell, David/Valentine, Gill (1995): Introduction: Orientations. In: Bell, D./Valentine, G. (Hrsg.): Mapping Desire. Geography of Sexualities. London: Routledge, S. 127.

Bütow, Birgit/Eckert, Lena/Teichmann, Franziska (2016): Fachkulturen als Ordnungen der Geschlechter. Praxeologische Analysen von Doing Gender in der akademischen Lehre. Opladen: Budrich.

Eckert, Lena (2014): Das Konzept des Lehrens mit Epistemologie zur Vermittlung von Gender als Querschnittsthema in der Hochschullehre. Ein lernendes Projekt. In: Freiburger Zeitschrift für Geschlechterstudien 20, 1, S. 47-62. https://doi.org/10.3224/fzg.v20i1.16343

Faulstich-Wieland, Hannelore/Wesseloh, Bianka (2016): Geschlechterverhältnisse. In: Koller, H.-C. et al. (Hrsg.): Datenreport Erziehungswissenschaft 2016. Schriften der Deutschen Gesellschaft für Erziehungswissenschaft (DGfE). Opladen: Budrich, S. 191-210. 
Ferfolja, Tania/Robinson, Kerry H. (2004): Why anti-homophobia education in teacher education? Perspectives from Australian teacher educators. In: Teaching Education 15, 1, S. 9-25. https://doi.org/10.1080/1047621042000179961

Fritzsche, Bettina/ Hartmann, Jutta/ Schmidt, Andrea/ Tervooren, Anja (Hrsg.). (2001): Dekonstruktive Pädagogik: Erziehungswissenschaftliche Debatten unter poststrukturalistischen Perspektiven. Wiesbaden: VS. https://doi.org/10.1007/978-3-663-09575-0

Gorski, Paul C./Davis, Shannon N./Reiter, Abigail (2013): An examination of the (in)visibility of sexual orientation, heterosexism, homophobia, and other LGBTQ concerns in U.S. multicultural teacher education coursework. In: Journal of LGBT Youth 10, 3, S. 224-248. https://doi.org/10.1080/19361653.2013.798986

Hartmann, Jutta (2002): Vielfältige Lebensweisen: Dynamisierung in der Triade Geschlecht - Sexualität - Lebensform. Kritisch-dekonstruktive Perspektiven für die Pädagogik. Forschung Erziehungswissenschaft, Band 157. Opladen: Leske + Budrich.

Helsper, Werner (1996): Antinomien des Lehrerhandelns in modernisierten pädagogischen Kulturen. Paradoxe Verwendungsweisen von Autonomie und Selbstverantwortlichkeit. In: Combe, A./Helsper, W. (Hrsg.): Pädagogische Professionalität. Untersuchungen zum Typus pädagogischen Handelns. Frankfurt am Main: Suhrkamp, S. 521-569.

Helsper, Werner/Tippelt, Rudolf(2011): Ende der Profession oder Professionalisierung ohne Ende? Zwischenbilanz einer unabgeschlossenen Diskussion. In: Helsper, W./Tippelt, R. (Hrsg.): Pädagogische Professionalität. Weinheim: Beltz, Zeitschrift für Pädagogik Beiheft, 57, S. 268-288.

Henningsen, Anja/Tuider, Elisabeth/Timmermanns, Stefan (2016): Einleitung: widersprüchliche Gleichzeitigkeit und Sexualpädagogik in der Kontroverse. In: Henningsen, A./Tuider, E./Timmermanns, S. (Hrsg.): Sexualpädagogik kontrovers. Weinheim: Beltz Juventa, S. 7-16.

Huch, Sarah/Lücke, Martin (2015): Sexuelle Vielfalt im Handlungsfeld Schule. Ein Vorwort. In: Huch, S./Lücke, M. (Hrsg.): Sexuelle Vielfalt im Handlungsfeld Schule: Konzepte aus Erziehungswissenschaft und Fachdidaktik. Pädagogik. Bielefeld: transcript, S. 7-12. https://doi.org/10.14361/9783839429617

Ihsen, Susanne (2013): Abschlussbericht des BMBF-Verbundprojektes „LeWI - Einstellungen von Lehrenden zur Lehre, Studienerfolg und Wirksamkeit von Interventionen zugunsten guter Lehre“. München: Technische Universität München.

Jäckle, Monika/ Eck, Sandra/ Schnell, Meta/ Schneider, Kyra (2016): Doing Gender Discourse: Subjektivation von Mädchen und Jungen in der Schule. Wiesbaden: VS Verlag für Sozialwissenschaften.

Klein, Uta/Rebitzer, Fabian A. (2012): Diskriminierungserfahrungen von Studierenden: Ergebnisse einer Erhebung. In: Heitzmann, D./Klein, U. (Hrsg.): Diversity konkret gemacht: Wege zur Gestaltung von Vielfalt an Hochschulen. Diversity und Hochschule. Weinheim: Beltz Juventa, S. 120-137.

Kleiner, Bettina (2015): subjekt bildung heteronormativität: Rekonstruktion schulischer Differenzerfahrungen lesbischer, schwuler, bisexueller und trans*Jugendlicher. Studien zu Differenz, Bildung und Kultur, Band 1. Opladen: Budrich.

Klinger, Sabine (2014): (De-)Thematisierung von Geschlecht: Rekonstruktionen bei Studierenden der Erziehungs-und Bildungswissenschaften. Opladen: Budrich. 
Klocke, Ulrich (2012): Akzeptanz sexueller Vielfalt an Berliner Schulen: Eine Befragung zu Verhalten, Einstellungen und Wissen zu LSBT und deren Einflussvariablen. Berlin: Senatsverwaltung für Bildung, Jugend und Wissenschaft.

Krell, Claudia/Oldemeier, Kerstin (2016): I am what I am? - Erfahrungen von lesbischen, schwulen, bisexuellen, trans* und queeren Jugendlichen in Deutschland. In: Gender - Zeitschrift für Geschlecht, Kultur und Gesellschaft 8, 2, S. 46-64. https://doi.org/10.3224/gender.v8i2.23733

KMK [Sekretariat der Ständigen Konferenz der Kultusminister der Länder in der Bundesrepublik Deutschland] (2000 [1980]): Empfehlung zur Förderung der Menschenrechtserziehung in der Schule. Verfügbar unter https://www.kmk.org/filead$\mathrm{min} /$ Dateien/veroeffentlichungen_beschluesse/1980/1980_12_04-Menschenrechtserziehung.pdf

Küpper, Beate/Klocke, Ulrich/Hoffmann, Lena-Carlotta (2017): Einstellungen gegenüber lesbischen, schwulen und bisexuellen Menschen in Deutschland: Ergebnisse einer bevölkerungsrepräsentativen Umfrage. Baden-Baden: Nomos.

Mills, Carmen/Ballantyne, Julie (2016): Social justice and teacher education: A systematic review of empirical work in the field. In: Journal of Teacher Education 67, 4, S. 263-276. https://doi.org/10.1177/0022487116660152

Nzimande, Nomkhosi (2015): Teaching pre-service teachers about LGBTI issues: Transforming the self. In: Agenda: Empowering women for gender equity 29, 1, S. 74-80. https://doi.org/10.1080/10130950.2015.1010299

Pongratz, Ludwig A. (2013): Unterbrechung: Studien zur Kritischen Bildungstheorie. Opladen: Budrich.

Robinson, Kerry H./Ferfolja, Tania (2008): Playing it up, playing it down, playing it safe: queering teacher education. In: Teaching and Teacher Education 24, 4, S. 846-858. https://doi.org/10.1016/j.tate.2007.11.004

Rommelspacher, Birgit (1998): Dominanzkultur: Texte zu Fremdheit und Macht. 2. Aufl. Berlin: Orlanda-Frauenverlag.

Schmidt, Sandra J./ Chang, Shih-pei/ Carolan-Silva, Aliah/ Lockhart, John/ Dorothea Anagnostopoulos, Dorothea (2012): Recognition, responsibility and risk: Pre-service teachers' framing and reframing of lesbian, gay, and bisexual social justice issues. In: Teaching and Teacher Education 28, 8, S. 1175-1184. https://doi.org/10.1016/j.tate.2012.07.002

Schmidt, Friederike/Schondelmayer, Anne-Christin/Schröder, Ute B. (2015): Selbstbestimmung und Anerkennung sexueller und geschlechtlicher Vielfalt. Lebenswirklichkeiten, Forschungsergebnisse und Bildungsbausteine - Einleitung. In: Schmidt, F./Schondelmayer, A.-C./Schröder, U. B. (Hrsg.): Selbstbestimmung und Anerkennung sexueller und geschlechtlicher Vielfalt. Wiesbaden: Springer VS, S. 9-22.

Smolen, Lynn A. et al. (2006): An empirical study of college of education faculty's perceptions, beliefs, and commitment to the teaching of diversity in teacher education programs at four urban universities. In: The Urban Review 38, 1, S. 45-61. https://doi.org/10.1007/s11256-005-0022-2

Unger, Hella von/Block, Martina/Wright, Michael T. (2007): Aktionsforschung im deutschsprachigen Raum. Discussion Papers / Wissenschaftszentrum Berlin für Sozialforschung, Forschungsschwerpunkt Arbeit, Sozialstruktur und Sozialstaat, Forschungsgruppe Public Health. Berlin. 
Villegas, Ana María/Lucas, Tamara (2002): Preparing culturally responsive teachers: Rethinking the curriculum. In: Journal of Teacher Education 53, 1, S. 20-32. https://doi.org/10.1177/0022487102053001003

Voß, Heinz-Jürgen (2011): Geschlecht. Stuttgart: Schmetterling. 